major fascial points as follows: thoracolumbar fascia, sacroiliac joint, pelvis ligaments, rotator cuff; potential nerve compressions (e.g., arcade of Frochse, soleus arcade); nerve sheath surrounding nerves (sciatic nerve, brachial plexus) and vessels (thoracic outlet syndrome), smaller fascia, joint capsule thickening. We detected higher rates of motility, improvement postural balance and pain decrease, fewer sessions needed in patients after extensive protocol.

Conclusion: Fascia dry needling is accessible and effective method for myo-fascial pain treatment, may provide additional mechanical benefit and help to maintain treatment effect. Affected fascia can be considered as relevant trigger points, specific ultrasound symptoms should be validated.

REFERENCES

[1] Bubnov R Trigger Points Dry Needling Under Ultrasound Guidance for Low Back Pain Therapy. Comparative Study. Annals of the Rheumatic Diseases2015;74:624. http://dx.doi.org/10.1136/annrheumdis-2015-eular.2323

[2] Bubnov R, Kalika L, Babenko L. Dynamic ultrasound for multilevel evaluation of motion and posture in lower extremity and spine. Annals of the Rheumatic Diseases 2018;77:1699. http://dx.doi.org/10.1136/annrheumdis-2018-eular.3949

Disclosure of Interests: None declared

DOI: 10.1136/annrheumdis-2021-eular.3843

\section{POS1285 1 FOOT DISORDERS AND FALLS RISK IN OLDER PERSONS}

N. Fani ${ }^{1}$, E. Toulgui ${ }^{1}$, D. Khalifa ${ }^{2}$, S. Mtaoua ${ }^{3}$, W. Ouanes ${ }^{1}$, S. Jemni ${ }^{1}{ }^{1}$ Hôpital Sahloul, Physical Medicine and Rehabilitation, Sousse, Tunisia; ${ }^{2}$ Hospital Farhat Hached, Rhumatologie, Sousse, Tunisia; ${ }^{3} \mathrm{Hospital}$ Ibn El Jazzar, Physical Medicine and Rehabilitation, Kairouan, Tunisia

Background: Falls are major problems in older people, leading to serious morbidity and mortality. Many studies have identified intrinsic and extrinsic fall risk factors in order to develop preventive strategies and guidelines. There are few reports which studied the impacts of aging feet on falls [1].

Objectives: The present study aimed to explore foot musculoskeletal disorders and their relationship to falls in a healthy aging population.

Methods: This was a cross-sectional descriptive study involving the patients aged 65 years and over who were independent in self-care and walking followed at the physical medicine and rehabilitation department of the university hospital of sousse over 3 months. The analysed data focused on the epidemiological and clinical characteristics. Trained physicians evaluated health status, foot problems, and fall(s) history of all subjects. Walking performance was assessed using the 'Timed Get Up \& Go' test and the unipedal stance test was used for the assessment of balance. Footprints were taken from the standing position. Associated factors of foot disorders and falls were analyzed.

Results: There were 45 subjects: 18 men, 27 women with a mean age of 69.6 [6586 ] years. Foot deformities presented in $87 \%$ and were a significantly associated with walking performance or falls $(p=0.01, p=0.02)$. The most common foot musculoskeletal disorder were Halux valgus $31 \%$, followed by Mallet toe $15 \%$. Arch of foot classified by physical examination revealed that pes planus was presented in $35 \%$ of cases. There was a significant correlation between BMl and an arch index $(p=0.04)$. Foot problems remained significantly associated with impaired timed up and go test and the unipedal stance test. The causes of pain were plantar fasciitis, hallux valgus, callus, metatarsalgia, and inappropriate footwear. Falls were reported in 33.3 of patients. Falls were significantly associated with Hallux valgus, mallet toe and pain $(p<0.05)$.

Conclusion: Independent of the influence of age, gender, common conditions; foot problems have a significant impact on the ability to perform functional tasks integral to independent living.

\section{REFERENCES:}

[1] Menz HB, Lord SR: Foot problems, functional impairment, and falls in older people. J Am Podiatr Med Assoc 1999;89:458- 467. 3 Americ

Disclosure of Interests: None declared

DOI: 10.1136/annrheumdis-2021-eular.3918

\section{POS1286 \\ SHORT-TERM AND MID-TERM EFFICACY OF CAUDAL EPIDURAL STEROID INJECTIONS IN THE MANAGEMENT OF DEGENERATIVE SCIATICA}

D. Khalifa ${ }^{1}$, N. El Fani ${ }^{1}$, R. Moncer ${ }^{1}$, E. Toulgui ${ }^{1}$, W. Ouanes ${ }^{1}$, S. Jemni ${ }^{1}$

${ }^{1}$ Sahloul Hospital, Physical Therapy and Rehabilitation, Sousse, Tunisia

Background: Epidural steroid injections are largely used in the management of osteoarthritis-related sciatica. Three possible sites of injection are possible: the caudal through the sacral hiatus, the interlaminar and the transforaminal site. The caudal technique is known to be the most simple one. However, doubts still persist about this infiltration's efficacy.

Objectives: The aim of this work was to study the short-term and mid-term efficacy of caudal epidural steroid injections in patients suffering from sciatica related to a degenerative etiology and to study the determining factors of its efficacy.
Methods: A retroscpective, descriptive and monocentric study was conducted in Sahloul university hospital of Tunisia. Medical records of patients who suffered from sciatica due to disc herniation or spinal stenosis were analysed. Only patients who benefited from at least one caudal epidural steroid injection were included. Other etiologies were ruled out by CT-scan or MRI and laboratory tests. Efficacy of the injection was evaluated by the visual analog scale of pain (VAS) at the first week post infiltration, 3 months and 6 months later. The infiltration was considered effective if the difference of pain scoe by VAS was $\geq 50 \%$ compared to baseline's score. The presence of anxiety and depression was also tracked down with the hospital anxiety and depression scale. Data was collected and analysed using the statistical tool SPSS.20.

Results: Twenty-five patients were included. They were 7 males and 18 females. The mean age at diagnosis was $51.88 \pm 15.28$ years. Eleven patients had another osteoarthritis site. Five patients had previous back surgery: 2 dissectomies, 1 arthrodesis, and 2 laminectomies. Median duration of sciatica was 30 months. Sciatica was bilateral in $41.7 \%$ of the cases, impulsive during efforts in $52 \%$ of the cases and with claudication in $92 \%$ of the cases. The median VAS score at baseline was 7 out of $10(\min =4 ; \max =9)$. All patients had tried medical treatment using NSAIDS and painkillers, and physical therapy before prescribing the infiltration. The technique was similar in all patients: Lidocaine $1 \%$ was first injected at a median volume of $5 \mathrm{ml}$, followed by corticosteroids and finally a saline solution (median volume of $20 \mathrm{ml}$ ). The median number of epidural caudal injections was 3 injections $(\min =1 ; \max =3$ ). The caudal epidural steroid injections were effective in $60 \%$ of the patients at the first week, $56 \%$ in the cases at 3 months and $56 \%$ of the cases at 6 months. Factors associated with efficacy of the injection at the first week were greater total volume injected $(p=0.001)$, and greater saline solution volumes $(p=0.016)$. At 3 months, factors significantly associated with efficacy of the infiltration were having unilateral pain $(p=0.05)$, a positive straight leg raise test sign $(p=0.028)$, a lower anxiety score $(0.014)$ and a lower depression $(0.000)$ score. At 6 months, factors associated with efficacy were not having cervical osteoarthritis $(p=0.03)$, unilateral pain $(p=0.05)$, low anxiety $(p=0.014)$ and low depression $(p=0.001)$ scores and a higher number of steroid injections $(p=0.05)$ Conclusion: Caudal epidural steroid injections seem effective on the short-term and this efficacy is maintained till the mid-term. Greater volumes may help with pain by possible adhesiolyse-like mechanisms and having unilateral pain, positive straight leg raise sign, a higher number of injections, no anxiety or depression and no other osteoarthritis sites makes the infiltration more effective.

\section{REFERENCES:}

[1] Dincer U, Kiralp MZ, Cakar E, Yasar E, Dursan H. Caudal epidural injection versus non-steroidal anti-inflammatory drugs in the treatment of low back pain accompanied with radicular pain. Joint Bone Spine. 2007;5.

Disclosure of Interests: None declared

DOI: 10.1136/annrheumdis-2021-eular.3967

\section{POS1287 ANXIETY, DEPRESSION, FIBROMYALGIA SYNDROME AND PAIN INTENSITY IN PATIENTS WITH LOW BACK} PAIN

R. Dhahri ${ }^{1}$, A. Dghaies ${ }^{1}$, M. Slouma ${ }^{1}$, L. Metoui ${ }^{1}$, I. Gharsallah ${ }^{1}$, I. Dorgham ${ }^{2}$, R. Ayari ${ }^{2}$, Y. Mallat ${ }^{2}, \mathrm{~K}$. Amri ${ }^{2}{ }^{1}$ The Military Hospital of Tunis, Rheumatology, Tunis, Tunisia; ${ }^{2}$ The Military Hospital of Tunis, Traumatology-Orthopedic Department, Tunis, Tunisia

Background: Common low back pain (LBP) is a common health problem affecting 50 to $80 \%$ of working age adults resulting in significant personal, social and occupational impairment. Chronic pain has a negative impact on quality of life and psychological well-being.

Objectives: The aim of this study was to examine the relationship between anxiety, depression and pain intensity in patients with low back pain in a population made of military agents.

Methods: It was an analytical cross-sectional study including 50 patients with at least three months of LBP, in the department of rheumatology and orthopedics at the Military Hospital of Tunis between January 1st and March 31, 2020. Al patients had a standardized clinical assessment. Patients completed the «hospital anxiety and depression scale» (HADS) and the «Fibromyalgia Rapid Screen ing Tool» (FIRST) to evaluate psychological impact of chronic LBP. Pain intensity was assessed using a visual analog scale (VAS)

Results: The mean age of the patients was $41.9 \pm 8.4$ years and the sex ratio was 4.5 . LBP duration was 66.4 months. The mean lumbar visual analog scale (VAS) was $4.5 \pm 1.9$, and the root VAS was $2.6 \pm 2.5$. Neuropathic pain was found in $26 \%$ of patients. Abnormal level of anxiety and depression were found in $58 \%$ and $62 \%$ of the patients respectively. Out of them $20 \%$ and $26 \%$ were borderline abnormal for anxiety and depression respectively, while $38 \%(16.4 \%)$ and $36 \%$ were certainly abnormal for anxiety and depression respectively. Twelve percent of the patients had fibromyalgia syndrome associated with LBP as they had a FIRST score $\geq 5$. Positive correlations were found between lumbar VAS and HAD anxiety/depression scores $(r=0.35 ; p<0.01 / r=0.3 ; p=0.04)$. Positive correlations were also found between root VAS and HAD anxiety/depression $(R=0.38$; 
$P<0.01 / R=0.29 ; P=0.03)$. There was positive correlations between Fibromyalgia syndrome (FIRST scale) and lumbar VAS $(r=0.42 ; p=0.01)$ and root VAS $(R=$ $0.29 ; \mathrm{P}<0.01)$.

Conclusion: The results of this study showed that anxiety, depression and fibromyalgia syndrome are not only frequent with patient suffering from chronic LBP but they are also correlated to the pain intensity. As a consequence, assessment of and intervention in anxiety, depression symptoms and fibromyalgia syndrome is crucial in the provision of pain-relief nursing treatment in patients with low back pain.

Disclosure of Interests: None declared

DOI: 10.1136/annrheumdis-2021-eular.4086

\section{POS1288 \\ TUBERCULOUS SPONDYLODISCITIS IN ELDERLY: EPIDEMIOLOGY, CLINICAL, FEATURES, TREATMENT AND OUTCOMES}

M. Mrabet ${ }^{1}$, S. Boussaid ${ }^{1}$, S. Jemmali ${ }^{1}$, H. Sahli ${ }^{1}$, H. Ajlani ${ }^{1}$, E. Cheour ${ }^{1}$, S. Rekik ${ }^{1}$, M. Elleuch ${ }^{1} .{ }^{1}$ Rabta Hospital, Rheumatology, Tunis, Tunisia

Background: Tuberculosis is still endemic all over the world. The incidence of tuberculous spondylodiscitis (TS) is steadily increasing. Clinical features and outcomes of this affection are various and depending on various parameters, including age.

Objectives: Our objective was to explore the differences in presentation and the results of further investigations and the prognosis of TS between young and elderly subjects.

Methods: We conduct a retrospective and descriptive study in a single rheumatology department. Data were collected from files of patients hospitalized in the past 20 years $(2000-2020)$ who have been diagnosed with TS. We carried out a comparative study concerning the clinical biological, imaging features and outcomes between young subjects and subjects aged over 65 years.

Results: Fifty-two cases of TS were collected (37F/15M). The mean age of the population was 55.21 years \pm 17.79 [19-91]. Thirty-three patients $(69.2 \%)$ were classified as young versus 16 elderly patients (30.8\%), with female predominance in both groups $(69.4 \%$ and $75 \%$ respectively, $p=0.57)$. Young subjects was more frequently vaccinated (88.9\%) by Bacillus Calmette-Guérin (BCG) $(p<0.001)$. A delayed diagnosis was noted in both groups $(p=0.24)$. Lumbar spine involvement was the most common $(57.7 \%)$. In the two age ranges, the onset of the disease was progressive $(p=0.22)$, characterized by segmental spine stiffness ( $p=0.57)$ and lumbar pain with general signs $(p=0.27)$, such as: impaired general condition, fever, night sweats and weight loss. Biological inflammatory syndrome and normochrome normocytic anemia were encountered in both cases $(p=0.08$ and $p=0.2$, respectively). Standard X-rays and Computed tomography were more performed in young subjects $(94.4 \%$ and $69.4 \%$, respectively; $p<0.001)$, unlike magnetic resonance imaging which was more common in elderly subjects but with no statistically significant difference $(p=0.22)$. Disc pinch, erosion of vertebral plateaus and vertebral collapse were the major signs $(82.7 \%, 65.4 \%$ and $67.3 \%$, respectively). Clinical, biological and imaging arguments contributed to positive diagnosis in both groups $(p=0.24)$. Common medical treatment was anti-tuberculosis: Isoniazid $(H)$, Rifampicin $(R)$, Pyrazinamide $(Z)$, Ethambutol $(E)$ and physical treatment such as immobilization witch was more common in the eldery $(56.3 \%, p=0.16)$. The evolution of the disease was characterized by a clear improvement of young subjects during the second week of treatment $(p<0.001)$. A more frequent clinical improvement in older subjects was during the fourth week but with no statistically significant difference $(p=0.13)$. The occurrence of immediate complications was more frequent in the elderly $(p=0.23)$ with a predominance for drug complications (56.3\%) such as: hepatic cytolysis (12.5\%), hyperuricemia (18.8\%) and major intolerance to anti-tuberculosis (18.8\%).

Conclusion: TS is a frequent condition that needs to be treated rapidly. the clinical presentation of TS in the elderly is less noisy which leads to more frequent complications and mortality.

Disclosure of Interests: None declared

DOI: 10.1136/annrheumdis-2021-eular.4129

\section{POS1289 CHRONIC LOW BACK PAIN: THERAPEUTIC STRATEGIES AND CLINICAL OUTCOMES IN A MILITARY POPULATION}

R. Dhahri ${ }^{1}$, A. Dghaies ${ }^{1}$, M. Slouma ${ }^{1}$, L. Metoui ${ }^{1}$, I. Gharsallah $^{1}$, I. Dorgham ${ }^{2}$, Y. Mallat ${ }^{2}$, R. Ayari ${ }^{2}$, K. Amri ${ }^{2} .{ }^{1}$ Military Hospital of Tunis, Rheumatology, Tunis, Tunisia; ${ }^{2}$ Military Hospital of Tunis, Orthopedics, Tunis, Tunisia

Background: Low back pain is an extremely common patient complaint. Most cases resolve quickly after the acute episode. However, a significant number of patients develop chronic low back pain; a persistent disabling condition. Patients suffer from unremitting pain and often become functionally impaired.

Objectives: The aim of this study is to describe the characteristics of chronic LBP, physical examination abnormalities, treatment strategies and the impact of LBP on the professional life of the patients.
Methods: It was a an analytical cross-sectional study including 50 patients with at least three months of LBP, in the department of rheumatology and orthopedics at the Military Hospital of Tunis between January 1st and March 31, 2020. All patients had a standardized investigation and clinical assessment.

Results: The study included $80 \%$ of active military serving members and $20 \%$ of administrative officers. The mean age of the patients was $41.9 \pm 8.4$ years and the sex ratio was 4.5 . Four patients were suffering from diabetes; two patients were suffering from high blood pressure. All the patients were suffering from chronic LBP lasting for an average of 66.4 months. LBP was associated with radicular pain in $78 \%$ of the cases. It was a unilateral radicular pain in $72 \%$ of the cases and bilateral in $28 \%$ of the cases. The main triggering factors were: carrying heavy loads in $98 \%$ of the cases, standing or sitting for long periods in $90 \%$ and $76 \%$ of the cases, tremors in $74 \%$ of the cases. Neuropathic pain was found in $26 \%$ of patients. Physical examination showed paravertebral muscle tenderness in $66 \%$ of the cases and slack abdominal muscles in $56 \%$ of the cases Assessement of range of flexion of the lumbar spine showed: fingertip to floor test was $18 \pm 12.2 \mathrm{~cm}[054 \mathrm{~cm}]$, schober test was $+3.8 \pm 1.2 \mathrm{~cm}[16 \mathrm{~cm}]$. The extension of lumbar spine was painful in $80 \%$ of the cases. A trigger point was found in $28 \%$ of the cases. Lasegue sign was positive in $18 \%$ of the cases. Leri's test was positive in $8 \%$ of the cases. Required treatments during the last episode of LBP were: Paracetamol $(62 \%)$, nonsteroidal antiinflammatory drugs $(26 \%)$, tramado $(4 \%)$, myorelaxant (4\%) and pregabalin (2\%). Half of the patients needed functional rehabilitation. Forty percent of the patients reported improvement; $46 \%$ of them reported improvement then recurrence of the pain, $8 \%$ of them reported no improvement and $4 \%$ reported worsening of the symptoms. Thirty six percent of the patients needed an average of 21 days of leave and $35 \%$ of them needed exemption from work for LBP problems. One patient needed an outplacement from his original work and one patient needed an early retirement.

Conclusion: Chronic low back pain can cause significant functional disability and commonly becomes frustrating for both patients and physicians to cope with and treat. There is still no consensus on the best way to manage chronic low back pain, and clinical guidelines are scarce. A combination of pharmacological agents and non-pharmacological methods is the most appropriate therapeutic regimen.

Disclosure of Interests: None declared

DOI: 10.1136/annrheumdis-2021-eular.4149

\section{\begin{tabular}{|l|l}
\hline POS1290 CYTOKINE BIOMARKERS IN COMMON LOW BACK \\
\hline
\end{tabular} PAIN}

R. Dhahri ${ }^{1}$, A. Dghaies ${ }^{1}$, M. Slouma ${ }^{1}$, L. Metoui ${ }^{1}$, I. Gharsallah ${ }^{1}$, I. Dorgham ${ }^{2}$, R. Ayari ${ }^{2}$, Y. Mallat ${ }^{2}$, K. Amri ${ }^{2}$, A. Tezeghdenti ${ }^{3}$, W. Dkhili ${ }^{3}$, R. Kochkar ${ }^{3}$, E. Ghazouani ${ }^{3} .{ }^{1}$ Military Hospital of Tunis, Rheumatology, Tunis, Tunisia; ${ }^{2}$ Military Hospital of Tunis, Orthopedics, Tunis, Tunisia; ${ }^{3}$ The Military Hospital of Tunis, Immunology, Tunis, Tunisia

Background: Common low back pain (LBP) is a common health problem affecting 50 to $80 \%$ of working age adults. It is one of the common and costly health problems in Tunisia. Actually, the role of the immune response and inflammatory cytokines in the pathogenesis of chronic pain has been of growing interest.

Objectives: The aim of this study was to assess whether pro and anti-inflammatory cytokines could be detected in serum in patients with LBP compared with healthy subjects and whether they could be related to pain severity and to clinical findings. Methods: It was a an analytical cross-sectional study including 50 patients with at least three months of LBP, in the department of rheumatology, orthopedics and immunology at the Military Hospital of Tunis between January 1st and March 31, 2020. All patients had a standardized clinical assessment.

Levels of serum cytokines IL-6, IL-8, IL-1 $\beta$ and TNF- $\alpha$, were measured using the chimiluminescence technique. Serum concentration of IL-10 was assayed by the enzyme-linked immunosorbent assay technique (ELISA). The normal levels of cytokines were determined in 50 healthy controls.

Results: The mean age of the patients was $41.9 \pm 8.4$ years and the sex ratio was 4.5. LBP duration was 66.4 months. The mean lumbar visual analog scale (VAS) was $4.5 \pm 1.9$, and the root VAS was $2.6 \pm 2.5$. Neuropathic pain was found in $26 \%$ of patients. The average BMI was $27 \pm 3.7 \mathrm{~kg} / \mathrm{m}^{2}$. Only serum level of IL-8 was significantly higher in subjects with LBP compared to healthy controls ( $p$ $<10-3)$. IL-1 $\beta$ was indetectable in both patients and controls. Positive correlations were found between IL-8 levels and anxiety/functional scores $(r=0.3 ; p=0.02$ $r=0.3 ; p=0.04$ ). IL-6 was positively correlated with BMI, and negatively correlated with the Schober test. No correlations were found between serum levels of IL-6, IL-8, IL-10, TNF- $\alpha$ and pain intensity (VAS), neuropathic pain (DN4), fibromyalgia (FIRST), depression (HAD) and various radiological data.

Conclusion: Interleukin-8 is a biomarker of common low back pain and correlate with anxiety and functional disability. These results suggest that IL-8 may be a therapeutic target to reduce chronic back pain and reduce the social and profession impact.

Disclosure of Interests: None declared

DOI: 10.1136/annrheumdis-2021-eular.4205 